\title{
On the Importance of Flow Direction in Business Process Models
}

\author{
Kathrin Figl and Mark Strembeck \\ Institute for Information Systems and New Media, \\ WU - Vienna University of Economics and Business, Welthandelsplatz 1, Vienna, Austria \\ \{kathrin.figl,mark.strembeck\}@wu.ac.at
}

Keywords: $\quad$ Model Layout, Reading Direction, Flow Direction, Business Process Models.

\begin{abstract}
In today's modeling practice we can observe a convention to model business processes from left-to-right or from top-to-bottom. Even though the choice of flow direction changes the visual appearance of a process model significantly, this convention is barely discussed by standard documents and modeling guidelines. In addition, most recommendations related to the flow direction are neither based on scientific claims nor on empirical evidence regarding their effectiveness in terms of readability. In this position paper, we discuss the importance of process model flow direction from a scientific viewpoint. In particular, we give a comprehensive overview of theoretical perspectives which offer explanations why a left-to-right flow direction for process models should be superior to other directions.
\end{abstract}

\section{INTRODUCTION}

Over the past decades business processes have developed into an essential means for the specification of the operational procedures in business companies and other professional organizations (see, e.g., Weske, 2007). Therefore, such processes do also directly affect the software systems that need to support the corresponding process flows.

In recent years, so called process-aware information systems (PAIS) emerged (see, e.g., Dumas et al., 2005) that facilitate the definition, execution, and monitoring of process flows. However, real-world process descriptions may become fairly complex. For example, a process flow may include sequential as well as parallel task executions. In addition, we often have loops where certain tasks are performed repeatedly. Moreover, a process description has to consider certain conditions that determine which of several alternative tasks needs to be performed in a certain situation.

Thus, in order to correctly define the corresponding process flows and communicate them to the different stakeholders, we need an expressive and comprehensible means to illustrate process descriptions. In this context, different graphical process modeling languages emerged, each of which provides a customized symbol set. A number of recent publications investigated the impact of different graphical symbols on process model comprehension (see, e.g., Figl et al., 2013a, Figl et al., 2013b). However, aside from symbol set design, a number of additional notational factors exist that may influence process model comprehension (see, e.g., Mendling et al., 2012, Reijers et al., 2011).

One of the comprehension factors that has not been intensively investigated yet is the modeling direction. In principle, process flows can be modeled from the left-hand side to the right-hand side or vice versa. Or they can be modeled from top-to-bottom or vice versa. For instance, the example models from the BPMN (Business Process Model and Notation) standard (Object Management Group, 2013a) and the example models of activity diagrams from the UML standard (Object Management Group, 2013b) are typically modeled from left-to-right. Other modeling directions are rather uncommon.

Figure 1 shows an excerpt of four process models, which are structurally and semantically equivalent, but use different flow directions.

While other layout factors of models (Effinger et al., 2011, Schrepfer et al., 2009) and their relationship to model understanding have already been investigated, to the best of our knowledge no theoretical discussion of flow direction has so far been undertaken. To fill this research gap, this paper presents a cumulative body of relevant knowledge and discusses the theoretical impact of different flow directions on process model comprehension. 


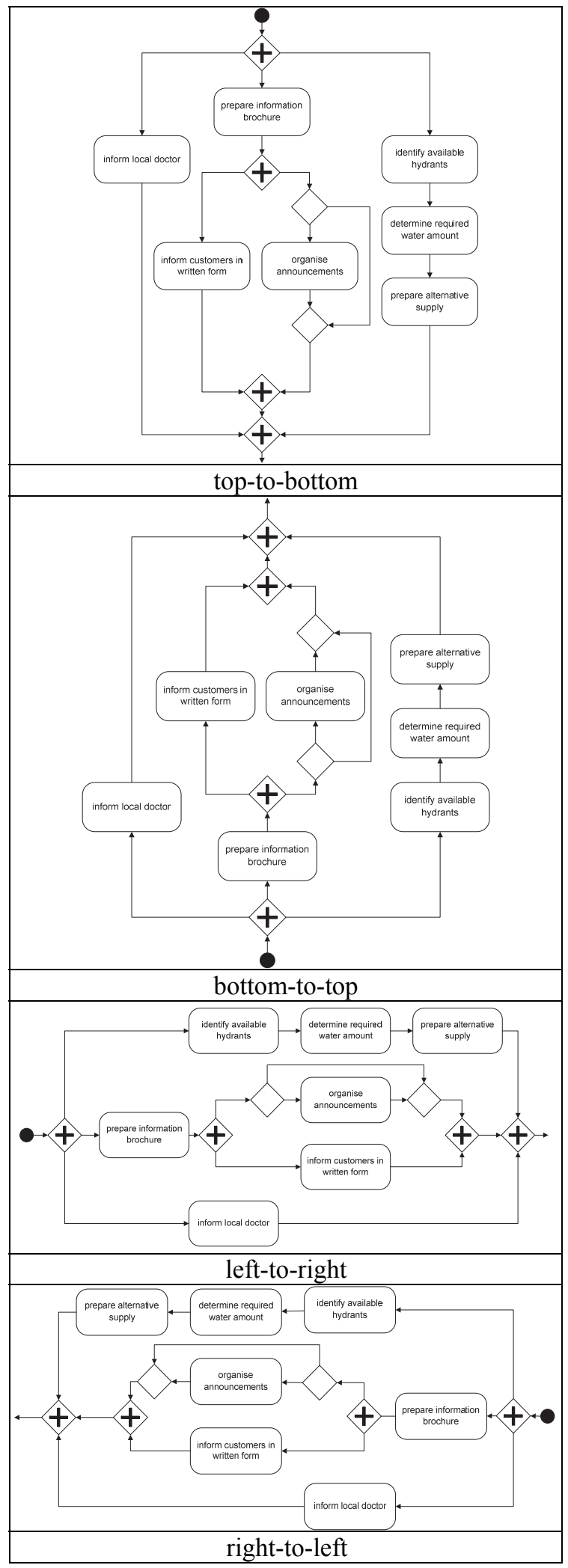

Figure 1: Detail of a BPMN process model in different flow directions.

\section{MODELLING STANDARDS AND CONVENTIONS}

Basic information about recommended flow directions in process models can be found in standard documents or in scientific publications on such notations. Petre (2006) describes such information as secondary notation - "things which are not formally part of a notation which are nevertheless used to interpret it, such as conventions (e.g., reading a circuit diagram left-to-right and topto-bottom)". Informal knowledge on the secondary notation can e.g. be found in guidelines, which, for instance, suggest to keep a uniform flow and edge direction in diagrams (Eichelberger and Schmid, 2009).

The BPMN standard document (Object Management Group, 2013a, p. 40) gives the following advice concerning modeling direction: "An incoming Sequence Flow can connect to any location on a Flow Object (left, right, top, or bottom). Likewise, an outgoing Sequence Flow can connect from any location on a Flow Object (left, right, top, or bottom). ...BPMN allows this flexibility; however, we also RECOMMEND that modelers use judgment or best practices in how Flow Objects should be connected so that readers of the Diagrams will find the behavior clear and easy to follow. This is even more important when a Diagram contains Sequence Flows and Message Flows. In these situations it is best to pick a direction of Sequence Flows, either left to right or top to bottom, and then direct the Message Flows at a $90^{\circ}$ angle to the Sequence Flows. The resulting Diagrams will be much easier to understand."

Thus, the BPMN standard document suggests using either a left-to right or top-to-bottom flow direction for modeling the sequence flow of a process model. However, the standard gives no specific rationale why these flow directions should be superior to others and does not clarify whether left-to-right or top-to-bottom is actually better suited for modeling the process flow. In the following, we will discuss the use of left-to-right or top-to-bottom orientations from several theoretical angels.

\section{READING DIRECTION IN DIAGRAMS AND CULTURAL INFLUENCE}

Petre (1995, p. 293) characterizes readers of graphical notations to be "more like the reader of a 
technical manual than the viewer of a painting: a deliberate reader, goal-directed and hypothesisdriven". This means that expectations and prior experience influence how people read diagrams and search for information. Thus, readers of process models actively use existing cues to find information; their main cue for determining the reading direction of the model is the direction of the arrows which represent connections in the process flow. Besides this visual hint on the flow direction, users follow typical reading strategies for diagrams. If not indicated otherwise, the usual reading direction for diagrams is similar to the written language: for example English-speaking individuals expect to read diagrams from left-to-right and from top-to-bottom (Winn, 1983, Gillespie, 1993). This is due to a strong cultural influence of the direction of written language for reading and drawing direction in general. For instance English-speaking children draw temporal concepts and call out names of objects from left-to-right, whereas right-to-left was dominant for Arabic and Hebrew-speaking children (Tversky et al., 1991).

Nordbotton and Crosby (1999) provide empirical evidence for reading strategy in the area of data models with eye tracking technology. On average, $60 \%$ of their participants followed a text-like reading strategy from left-to-right and top-to-bottom, $40 \%$ an image-like reading strategy (starting in the center followed by scanning in different directions).

Winn (1982, p. 80) states that "diagrams not arranged in this logical sequence would lead to difficulty in information processing and to less learning.". This is because people anticipate certain characteristics in diagrams according to previously learned diagram schemas, and understanding is easier if diagrams match these expectations (Winn, 1983).

Indeed, Winn (1982) was able to demonstrate that for native English speakers it is more difficult to learn sequences in reversed-order (right-to-left) than in normal-order (left-to-right) diagrams. Similarly, research on flowcharts has shown, that directional orientation influences problem solution quality, time taken to view the charts and time taken to solve the problems (Krohn, 1983). Participants performed best when orientation of flowcharts was consistent with reading direction (best results for left-to-right, second-best results for top-to-bottom and worst results for right-to-left flowcharts). They made fewer errors and needed less time. The phenomenon that consistency between direction in the learning material and expected reading direction supports reasoning was also confirmed in another context by
Harsel et al. (1987). They found that performance on inductive reasoning tasks was higher when material was presented in the direction of written language (the Japanese sample performed better with the vertical version, while the Australian sample performed better with the horizontal version).

However, subjects can develop "reversed diagram" schemas when working with reversed diagrams (Winn, 1983). Winn found evidence for this phenomenon by investigating eye-movements in a study with right-to-left reversed diagrams. At first, participants performed worse in information searching tasks than participants with left-to-right diagrams, because they started to search information in the upper left quadrant of the diagram. Though, after four trials they started looking at the bottom left quadrant which contained more useful information. Winn concludes that if diagrams contradict usual schemas, they are more difficult to understand and provoke more errors in information search tasks, but an appropriate perceptual strategy can be obtained after time.

\section{CULTURAL CONVENTIONS OF USING SPATIAL ORIENTATIONS AND SEMANTIC ASSOCIATIONS OF SPATIAL ORIENTATIONS}

Understanding complex processes demands logical thinking and reasoning. We know from cognitive psychology that humans use internal spatial representations when they solve logical problems, even for nonspatial and abstract problems (Handel et al., 1968). In that context, a variety of studies have revealed that humans use specific orientations (left, right, top, bottom) for abstract semantic concepts and that some associations are more likely than others.

For instance, if participants get two premises as "Tom is better than Bill" and "Bill is better than Mike" and then have to answer the question "Is Tom better than Mike?" they mentally imagine the names on a vertical axis for problem solving. In a betterworse relation they would imagine the "better" individual above the "worse" individual, so "better than" proceeds from top-to-bottom and "worse than" from bottom-to-top (De Soto et al., 1965, p. 517). Similarly, persons consistently spatially assign topto-bottom in a cognitive space for relation words as "father-son" and "more-less" (Handel et al., 1968, p. 354). There is a strong association between "more, 
better, and good" with upward and "less, worse, and bad" with downward (Tversky et al., 1991, p. 518). This can also be seen in linguistic metaphoric expressions such as "She is feeling down today" or in the fact that increases are usually displayed from bottom-to-top in graphics (Tversky et al., 1991).

When turning to relation pairs relevant to the context of process modeling, the scientific literature reveals that there is a clear preference to assign "earlier-later" to left-to-right followed by top-tobottom and to assign "cause-effect" to top-to-bottom and left-to-right (Handel et al., 1968, p. 354). According to Winn (1982) research has not yet given clear answers on how diagrams could best convey information about "the sequence of concepts", but following the above arguments, it would be most naturally to design process models from left-to-right, and top-to-bottom is likely to be the second best option. These orientations would also be consistent with a readers' mental visual orientation associated with the direction of a process flow.

While it is not clear from the literature whether these internal associations between semantic concepts and spatial orientations are actually caused by conventions in visual representations (as diagrams, tables, or text) or vice versa, humans have chosen to use these conventions, because they seem more natural, and a variety of examples demonstrate that specific semantic concepts are used predominantly with specific orientations. For instance, when looking at how temporal relations are represented in every-day life it is interesting to note that often top-to-bottom orientation is used (e.g. calendars, school schedules, programs, public transport schedules). In graphs, time is usually expressed from left-to-right on the horizontal axis (Tversky et al., 1991, p. 546).

\section{PRACTICAL AND NEUROPSYCHOLOGICAL ASPECTS OF SPATIAL ORIENTATIONS}

To complete our discussion of different theoretical perspectives, we now discuss factors that relate to spatial orientations which go beyond cultural conventions of direction in written language and visual representations.

First, there are also purely practical reasons accounting for why cultures have chosen specific writing directions. To give an example, independent of the horizontal or vertical as well as the left-right or right-left organization, pictographic and alphabetic writing systems are usually produced and read from top-to-bottom, probably because "the hand shouldn't cover what has just been written" (Tversky et al., 1991, p. 551).

Second, there also seem to be neuropsychological causes for a general human preference for left-to-right in the context of reading and writing. Such preferences for left-to-right orientation can be found in various incidents. For instance most adults, but also children who have not yet learned writing tend to draw pictures from leftto-right (Hufschmidt, 1985). Such findings would suggest that left-to-right preferences also have inborn aspects and are not only acquired. One reason behind the left-to-right preference could be the association of language with the left cerebral hemisphere which led to a "dominance of the left field of vision" (Hufschmidt, 1985). Recent research in the area of spatial processing for instance claims that there exists a spatial asymmetry in visual shortterm memory. Sala et al. (2010) showed that people remember objects in perception tasks better when they were positioned on the left than on the right. Attention seems to be focused on the left-hand side.

\section{HYPOTHESES}

Following from the theoretical discussion above, we will now advance propositions regarding the superiority of specific flow directions in regard to process model understandability. One of the essential arguments is that understanding a process model will be easier if its flow direction matches users' expectations (Harsel and Wales, 1987, Krohn, 1983, Winn, 1982). Such expectations are formed by the direction of written language and typical conventions used in visual representations (Tversky et al., 1991, Winn, 1983). Furthermore, humans associate specific semantic concepts with spatial orientations. In light of the above arguments, we specifically expect that left-to-right flow direction in a model is superior to other flow directions (top-tobottom, bottom-to-top, right-to-left) with respect to process model comprehension. This is because it is consistent with text reading direction, the association between semantic concepts as "earlier-later" and left-to-right (Handel et al., 1968) and a human preference for left-to-right orientation (Hufschmidt, 1985). 


\section{CONCLUSION}

This paper has presented a cumulative body of knowledge relevant to flow direction in diagrams and this integration of research streams denotes an important extension to the scientific discussion on layout choices for process models. From a theoretical perspective, advising left-to-right flow direction is beneficial. In this context, our position paper serves as a contribution to existing process model layout considerations and secondary notation research in general. We already designed a corresponding experiment and in our future work, we will conduct this experiment to examine the alleged superiority of the left-to-right flow direction for process model comprehension.

\section{REFERENCES}

De Soto, C. B., London, M., Handel, S., 1965. Social reasoning and spatial paralogic. Journal of Personality and Social Psychology, 2, 513-521.

Dumas, M., Van Der Aalst, W. M. P., Ter Hofstede, A. H. M. (eds.), 2005. Process Aware Information Systems: Bridging People and Software Through Process Technology, Hoboken, New Jersey: John Wiley \& Sons.

Effinger, P., Jogsch, N., Seiz, S., 2011. On a Study of Layout Aesthetics for Business Process Models Using BPMN. In: MENDLING, J., WEIDLICH, M. \& WESKE, M. (eds.) Business Process Modeling Notation. Springer Berlin Heidelberg.

Eichelberger, H., Schmid, K., 2009. Guidelines on the aesthetic quality of UML class diagrams. Information and Software Technology, 51, 1686-1698.

Figl, K., Mendling, J., Strembeck, M., 2013a. The Influence of Notational Deficiencies on Process Model Comprehension. Journal of the Association for Information Systems, 14.

Figl, K., Recker, J., Mendling, J., 2013b. A study on the effects of routing symbol design on process model comprehension. Decision Support Systems, 54, 11041118.

Gillespie, C. S., 1993. Reading Graphic Displays: What Teachers Should Know. Journal of Reading, 36, 35054.

Handel, S., Desoto, C. B., London, M., 1968. Reasoning and spatial representations. Journal of Verbal Learning and Verbal Behavior, 7, 351-357.

Harsel, Y., Wales, R., 1987. Directional preference in problem solving. International Journal of Psychology, 22, 195-206.

Hufschmidt, H.-J., 1985. Zeichnungsrichtung, Schreibrichtung und Blickfelddominanz. European Archives of Psychiatry and Clinical Neuroscience, 235, 76-81.
Krohn, G. S., 1983. Flowcharts Used for Procedural Instructions. Human Factors, 25, 573-581.

Mendling, J., Strembeck, M., Recker, J., 2012. Factors of Process Model Comprehension - Findings from a Series of Experiments. Decision Support Systems, 53, 195-206.

Nordbotten, J. C. \& Crosby, M. E., 1999. The effect of graphic style on data model interpretation. Information Systems Journal, 9, 139-155.

Object Management Group, 2013a. Business Process Model and Notation (BPMN) Version 2.0.2.

Object Management Group, 2013b. OMG Unified Modeling Language (OMG UML). In: GROUP, T. O. M. (ed.).

Petre, M. 1995. Why looking isn't always seeing: readership skills and graphical programming. Commun. ACM, 38, 33-44.

Petre, M., 2006. Cognitive dimensions 'beyond the notation'. Journal of Visual Languages \& Computing, 17, 292-301.

Reijers, H. A., Mendling, J., Dijkman, R. M., 2011. Human and automatic modularizations of process models to enhance their comprehension. Information Systems, 36, 881-897.

Sala, S. D., Darling, S., Logie, R. H., 2010. Items on the left are better remembered. The Quarterly Journal of Experimental Psychology, 63, 848 - 855.

Schrepfer, M., Wolf, J., Mendling, J., Reijers, H. A., 2009. The Impact of Secondary Notation on Process Model Understanding. In: The Practice of Enterprise Modeling, 2nd IFIP WG8.1 Working Conference (PoEM 2009) Stockholm, Sweden.

Tversky, B., Kugelmass, S., Winter, A., 1991. Crosscultural and developmental trends in graphic productions. Cognitive Psychology, 23, 515-557.

Weske, M., 2007. Business Process Management: Concepts, Languages, Architectures, Springer-Verlag.

Winn, W., 1982. The role of diagrammatic representation in learning sequences, identification and classification as a function of verbal and spatial ability. Journal of Research in Science Teaching, 19, 79-89.

Winn, W., 1983. Perceptual strategies used with flow diagrams having normal and unanticipated formats. Perceptual and Motor Skills, 57, 751-762. 